\title{
Transport and percolation in a low-density high-mobility two-dimensional hole system
}

\author{
M. J. Manfra ${ }^{1}$, E. H. Hwang ${ }^{2}$, S. Das Sarma ${ }^{2}$, L. N. Pfeiffer ${ }^{1}$, K. W. West ${ }^{1}$, and A. M. Sergent ${ }^{1}$ \\ ${ }^{1}$ Bell Laboratories, Alcatel-Lucent, Murray Hill, New Jersey, 07974 \\ 2 Condensed Matter Theory Center, Department of Physics, \\ University of Maryland, College Park, Maryland, 20742
}

\begin{abstract}
We present a study of the temperature and density dependence of the resistivity of an extremely high quality two-dimensional hole system grown on the (100) surface of GaAs. For high densities in the metallic regime $\left(p \gtrsim 4 \times 10^{9} \mathrm{~cm}^{-2}\right)$, the nonmonotonic temperature dependence $(\sim 50-300$ $\mathrm{mK}$ ) of the resistivity is consistent with temperature dependent screening of residual impurities. At a fixed temperature of $T=50 \mathrm{mK}$, the conductivity vs. density data indicates an inhomogeneity driven percolation-type transition to an insulating state at a critical density of $3.8 \times 10^{9} \mathrm{~cm}^{-2}$.
\end{abstract}

The low temperature conduction properties of an interacting two-dimensional (2D) electron gas in the presence of disorder remains a topic of fundamental interest and some controversy. It has been understood for some time that all non-interacting electronic states are localized in the presence of disorder at $T=0$ [1]. This model was challenged by the observation of Kravchenko and coworkers [2] of an apparent metal-to-insulator transition in Si MOSFETs. Subsequently a variety of models have been put forth [3, 4, 5, 6] to explain a wealth of experimental data. The 2D metal-to-insulator transition (MIT) has now been studied in a variety of semiconductor systems [2, 7, 8, 9, 10, 11, 12, 13, 14. Yet the question remains: does the transition from insulating to metallic behavior observed in certain $2 \mathrm{D}$ systems represent a quantum phase transition to a novel metallic state stabilized by interactions [5] or can the experimental data in the metallic regime be explained in terms of finitetemperature Fermi liquid behavior [4] ? The MIT in Si MOSFETs has been interpreted as evidence for quantum critical behavior [8]. However, recent experimental work [11, 15 in ultra-high mobility n-GaAs 2D systems has interpreted the MIT as a density inhomogeneity induced percolation transition.

In this Letter, we present a study of the transport properties of a clean two-dimensional hole system (2DHS) in the vicinity of the putative metal-to-insulator transition. Our carbon-doped 2DHSs display mobilities in excess of $2 \times 10^{6} \mathrm{~cm}^{2} /$ Vs at densities $\mathrm{p} \leq 3 \times 10^{10} \mathrm{~cm}^{-2}$, the highest ever achieved in a 2DHS in this density range. The use of extremely high mobility samples allows us to probe the regime of large $r_{s}\left(r_{s}=\mathrm{E}_{C} / \mathrm{E}_{F}\right.$, where $\mathrm{E}_{C}$ is the Coulomb interaction scale and $\mathrm{E}_{F}$ is the Fermi energy) in a clean system, where the effects of carrier-carrier interactions should be most apparent. Interestingly, we find that for high densities in the metallic regime the nonmonotonic temperature dependence of the resistivity is consistent with temperature dependent screening of residual impurities. At a fixed temperature of $T=50 \mathrm{mK}$, the conductivity vs. density data indicates a disorderinduced percolation-type transition to an insulating state at low density. While the critical density $\left(p_{c} \sim 3.8 \times 10^{9}\right.$ $\left.\mathrm{cm}^{-2}\right)$ and the interaction parameter $\left(r_{s} \sim 45\right)$ for our sample are among the lowest and highest ever respectively recorded in the 2D MIT literature, we find that the data are well explained within this simple framework. The fact that our critical density is similar to the critical density for 2D MIT found in ref. [11] suggests that the effective 2D MIT in GaAs electron and hole systems at experimentally accessible temperatures is a disorder-driven crossover phenomenon and not an interaction driven quantum phase transition, since the $r_{s}$ value for our hole system is four times larger than the corresponding $r_{s}$ in the electron system of ref. [11].

Our experiments utilize recently developed structures in which the 2DHS resides in a $20 \mathrm{~nm}$ GaAs/AlGaAs quantum well grown on the (100) surface of GaAs that has been modulation-doped with carbon [16]. A backgated Hall bar geometry is used in this experiment to tune the density from $2.9 \times 10^{10} \mathrm{~cm}^{-2}$ to $2.9 \times 10^{9} \mathrm{~cm}^{-2}$ in single sample. Cyclotron resonance studies of similarly grown carbon-doped quantum wells indicate that the hole effective mass is large, $m_{h} \sim 0.3 m_{e}$, where $m_{e}$ is the free electron mass 17. To give a comparison with other $2 \mathrm{D}$ systems studied in the literature, we mention that the 2D n-GaAs samples of refs. [10, 11] have an electron mobility of $5 \times 10^{6} \mathrm{~cm}^{2} /$ Vs at the carrier density of $3 \times 10^{10} \mathrm{~cm}^{-2}$ compared with our hole mobility of $2 \times 10^{6}$ $\mathrm{cm}^{2} / \mathrm{Vs}$ at the same density. Taking into account the effective mass difference $\left(m=0.07 m_{e}\right.$ for electrons in refs. [10] and [11] and $m \approx 0.3 m_{e}$ for holes in our samples) and remembering that mobility $\mu \propto \tau / m$, where $\tau$ is the transport relaxation time, we conclude that our $2 \mathrm{D}$ hole samples are comparable in quality to the corresponding 2D electron samples of refs. [10, 11] while $r_{s}$ is significantly larger. In this manner the data presented here probe a regime inaccessible to the experiments of refs. 10, 11].

Figure 1 displays magnetotransport at $T=50 \mathrm{mK}$. The exceptional quality of these samples is evident in the strength of the fractional quantum Hall state at $\nu=2 / 3$ at $\mathrm{B} \sim 1$ Tesla and the nascent features observed at $\nu=5 / 3$ and $4 / 3$ at even lower values of magnetic field. Figure 2 displays the temperature dependence of the resistiv- 


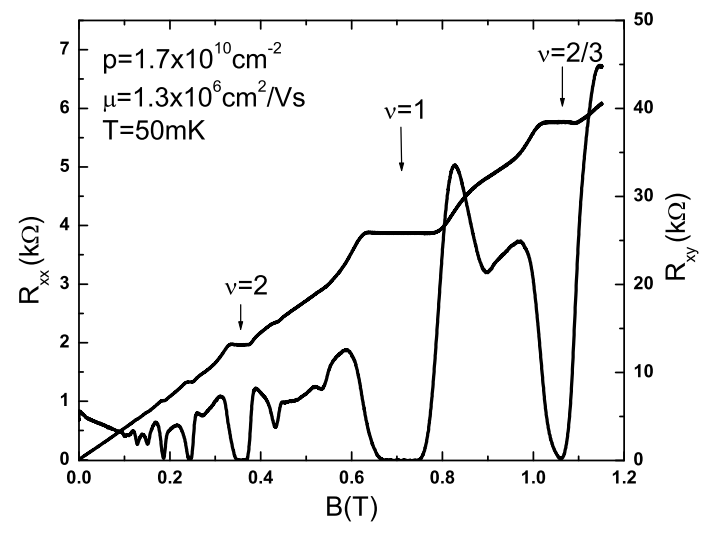

FIG. 1: Longitudinal and Hall resistance at zero gate bias. The fractional quantum Hall state at $\nu=2 / 3$ is fully developed at $\mathrm{B} \sim 1 \mathrm{~T}$ while weaker features at $\nu=5 / 3$ and $4 / 3$ are also visible.

ity at $\mathrm{B}=0$ for several values of density $\mathrm{p} \leq 9 \times 10^{9} \mathrm{~cm}^{-2}$. At all but the lowest densities, the temperature dependence is strongly nonmonotonic [18]. Starting from the high temperature regime $(T \sim 1 \mathrm{~K})$ the resistivity first increases with decreasing temperature, reaches peak value, and then decreases rapidly with further reduction in temperature. At $\mathrm{p}=9.0 \times 10^{9} \mathrm{~cm}^{-2}$, the low temperature drop in resistance is a factor of 2 . As the density is reduced this crossover point, where $d \rho / d T$ changes sign, moves to lower temperature, presumably tracking the reduction in the Fermi energy of our 2D system. The metallic behavior persists to very low density, at $\mathrm{p}=4.1 \times 10^{9} \mathrm{~cm}^{-2}$ $d \rho / d T$ still changes sign near $\mathrm{T}=50 \mathrm{mK}$. At $\mathrm{p}=2.9 \times 10^{9}$ $\mathrm{cm}^{-2}$, the lowest density accessed in our study, $\rho$ vs. $T$ is monotonic, suggesting that the sample is approaching an insulating regime. Yet at $\mathrm{p}=2.9 \times 10^{9} \mathrm{~cm}^{-2}$ the conductivity is not exponentially suppressed with decreasing temperature as expected for an insulating state, but rather, the conductivity displays a linear dependence on temperature (i.e. $\rho \propto T^{-1}$ ) in the $T=50-450 \mathrm{mK}$ range [19]. In this regime of low density, the Fermi temperature is as low as $T_{F} \sim 250 \mathrm{mK}$. The impact of carrier degeneracy and the temperature dependence of ionized impurity scattering must be considered as discussed below.

In order to understand the low temperature 2D transport properties in the metallic regime we have carried out a microscopic transport calculation using the Boltzmann theory [4]. In our calculation we assume that the 2D carrier resistivity is entirely limited by screened impurity scattering. We neglect all phonon scattering effects because our theoretical estimate shows phonon scattering to be negligible for $2 \mathrm{D}$ holes in GaAs structures in the $T<1 K$ regime of interest to us. Formally, this approach is well-controlled in the regime of small $r_{s}$ and $k_{F} l \gg 1$, where $k_{F}$ is the Fermi wavevector and $l$ is the mean free

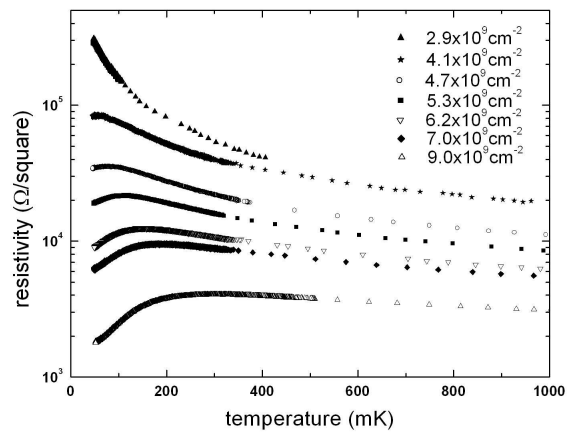

FIG. 2: Temperature dependence of the resistivity for 50 $\mathrm{mK} \leq \mathrm{T} \leq 1 \mathrm{~K}$ for densities ranging from $9.0 \times 10^{9} \mathrm{~cm}^{-2}$ to $2.9 \times 10^{9} \mathrm{~cm}^{-2}$.

path. While our Fermi liquid approach is not well controlled at high interaction, it has been proven to work at relatively large $r_{s}$ which substantiates its use in the high density, large conductance regime $k_{F} l>1$. We therefore mostly show our theoretical results in the $k_{F} l>1$ metallic regime except for the lowest density (dashed) curve in Fig. 3(a). We emphasize that by adjusting the charged impurity density and by including disorder effects in the screening function, it is possible to get reasonable quantitative agreement between our Boltzmann-Fermi liquid theory [Fig. 3(a)] and the experimental data (Fig. 2) even in the insulating low-density $k_{F} l<1$ regime [20]. We show our calculated results in Fig. 3 for various hole densities. In our calculation the overall resistivity scale depends on the unknown charge impurity density, but the qualitative trends in $\rho(T, p)$ arise from basic aspects of the underlying scattering mechanism. Our calculated resistivity, Fig. 3(a), shows a non-monotonicity (as seen in Fig. 2 of the experimental data) where it increases with $\mathrm{T}$ at first and then decreases with increasing $\mathrm{T}$ after going through a maximum at a density-dependent temperature $T_{m}$ where $d \rho / d T$ changes sign. This non-monotonicity is a non-asymptotic finite temperature "quantum-classical" crossover phenomenon, and arises from the competition between two contributions to transport [4], i.e. the temperature dependence of the dielectric screening function and the thermal averaging of the scattering rate. The screening effect decreasing with increasing temperature gives rise to increasing effective disorder with increasing $\mathrm{T}$, and hence increasing $\rho(T)$ with T. Based on the Boltzmann theory, however, in the higher temperature classical non-degenerate regime the thermal averaging of the scattering rate becomes quantitatively more important and shows stronger temperature dependence than screening, which gives rise eventually to a $\rho(T)$ decreasing with $\mathrm{T}$ for $T>T_{m}$. Thus, if the system is homogeneous and not strongly localized this crossover behavior appears in the transport measurements as can be seen in Fig. 2. We note that as the density is reduced significantly be- 


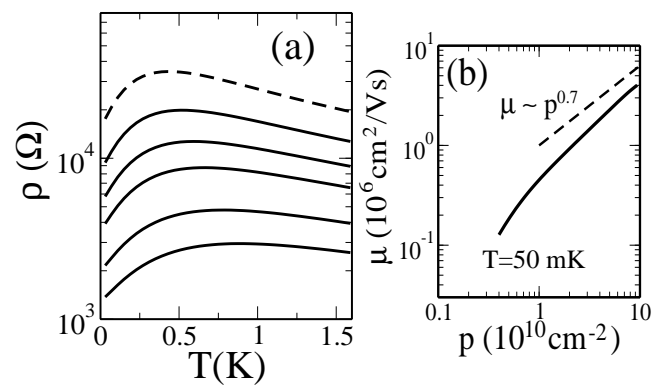

FIG. 3: (a) Calculated resistivities for p-GaAs quantum well system $(a=200 \AA)$ for various hole densities $p=0.4,0.5$, $0.6,0.7,0.9,1.1 \times 10^{10} \mathrm{~cm}^{-2}$ (from the top) with linearly screened charge impurity scattering. (The dashed curve is in the $k_{F} l<1$ regime.) (b) Calculated mobility as a function of hole density at $T=50 \mathrm{mK}$. The dashed straight line indicates the $\mu \sim p^{0.7}$ behavior. We only show mobility in the $k_{F} l>1$ regime.

low $p=9.0 \times 10^{9} \mathrm{~cm}^{-2}, k_{F} l \sim 1$ and direct comparison between theory and experment becomes more problematic. Nevertheless, we point out that the theory reproduces well all the qualitative features of the data in the metallic regime shown in Fig. 2. (1) An approximately (and upto) a factor of 2 increase in low-temperature $\rho(T)$ with increasing $T$; $(2)$ a maximum in $\rho(T)$ at a density dependent $T_{m}(p)$; (3) a "high-temperature" decrease of $\rho(T) \sim T^{-1}$ for $T>T_{m}$. One important difference between the 2D n-GaAs electron system [9, 10, 11] and our $2 \mathrm{D}$ p-GaAs hole system is the very strong metallic temperature dependence ( $\sim$ upto $200 \%$ ) we observe (cf. Fig. $2)$ in our hole sample versus the rather weak $(\sim 20 \%$ only) temperature dependence in the metallic phase of the $\mathrm{n}$-GaAs electron system [10, 11]. This strong (weak) metallic temperature dependence in the 2D hole (electron) GaAs system is easily explained by the screening theory 22] as arising from the much larger (by a factor four) dimensionless screening parameter $q_{T F} / 2 k_{F}$ in the hole system compared with the electron system due to the larger hole effective mass. In Fig. 3(b) we show the calculated mobility as a function of hole density at $T=50 \mathrm{mK}$. At high densities $\left(p \geq 1.0 \times 10^{10} \mathrm{~cm}^{-2}\right)$ the exponent $\alpha$ in $\mu \propto p^{\alpha}$ is about 0.7 , which is consistent with experiment (cf. Fig. 4) indicating that the screened charged impurity is the main scattering source.

In order to gain further insight into the transition to insulating behavior at low density we now focus on the density dependence of the conduction properties at fixed low temperature. Figure 4(a) shows the dependence of mobility on density at fixed temperature $\mathrm{T}=47 \mathrm{mK}$. While the data for all densities cannot be described by a single power law behavior, at densities above $\sim 2 \times 10^{10} \mathrm{~cm}^{-2}$ the mobility approaches an approximate power law behavior $\mu \sim p^{0.7}$ as seen in our theory. At low densities, $\mathrm{p} \leq 1 \times 10^{10} \mathrm{~cm}^{-2}$, the power law changes to a much stronger function of density, pointing to a change in the
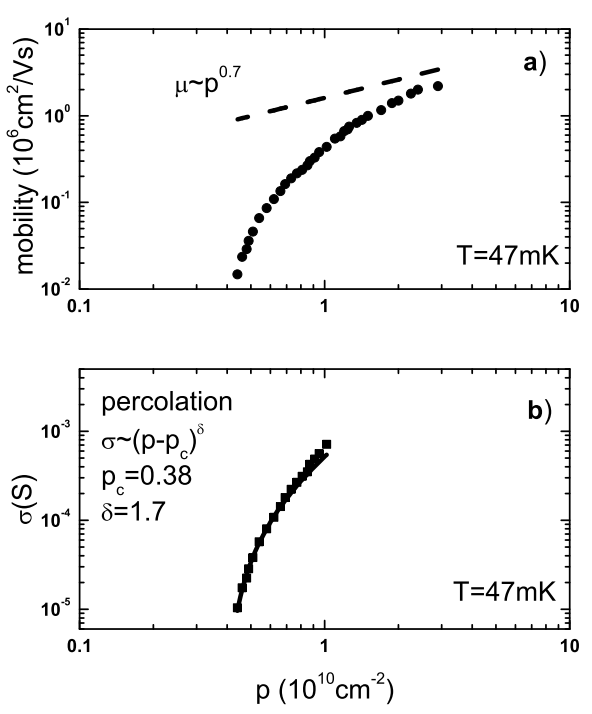

FIG. 4: (a) Mobility vs. density at fixed temperature $T=47$ mK. (b) Conductivity vs. density (solid squares) along with the fit generated assuming a percolation transition. The dashed line in (a) indicates the $\mu \sim p^{0.7}$ behavior.
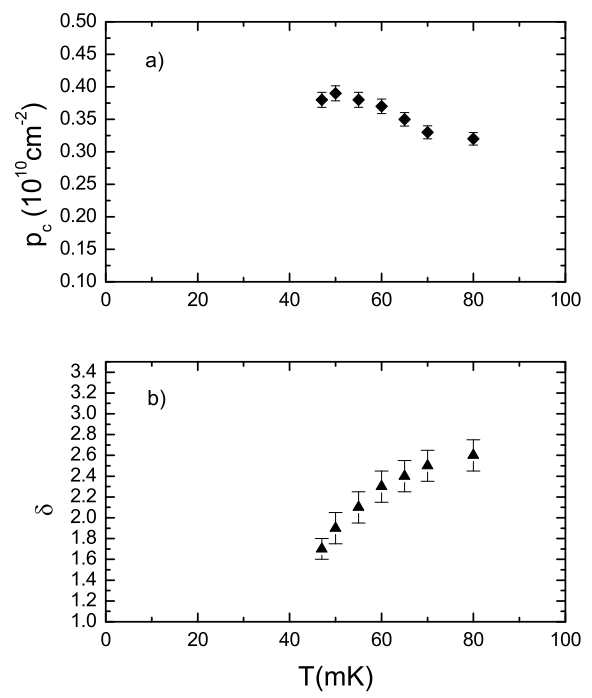

FIG. 5: a) Variation of the derived critical density $\mathrm{p}_{c}$ as a function of temperature. b) Variation of $\delta$ with temperature.

screening properties of the system at low carrier density. We emphasize that the quantitative and qualitative discrepencies between the lowest density data of Fig. 4a and the calculation of Fig. 3b indicate that the screening theory which captures the essential experimental features at higher densities does not fully describe the transition to insulating behavior.

For high mobility n-type GaAs, Das Sarma et al. 11] interpreted the density driven crossover from metallic 
conductivity to an insulating regime as a percolationtype transition. The importance of interactions as parameterized by $r_{s}$ for the MIT in GaAs remains an open question. In high mobility n-type $2 \mathrm{D}$ layers, the regime of $r_{s} \sim 10$ can be reached at low density. Due to the higher effective mass in our 2DHS, $r_{s}$ reaches approximately 45 at low density in this study. It is an interesting question if a percolation type analysis can also be applied to our ultra high mobility 2DHS in the regime of large $r_{s}$. We mention that there have also been various percolation analyses of 2D MIT in other n- and p-GaAs systems [14, 21] for low mobility (a factor of 10 lower than our mobility) systems where the temperature dependent metallic behavior is much weaker than what we see in our high-mobility system.

To describe the transition of the resistivity from metallic behavior to an apparent insulating behavior at lowest densities, we fit our data using a percolation transition model [22]. The physical motivation for a percolation transition is readily apparent. As the density of the 2DHS is reduced, the system can no longer fully screen the random disorder potential. The 2DHS breaks up into "puddles" of carriers isolated from one another by peaks in the disorder potential. Transport then takes place through percolating conduction paths in the disorder potential network. In the percolation model, the conductivity near the percolation threshold density is described by:

$$
\sigma(p)=A\left(p-p_{c}\right)^{\delta}
$$

where $\sigma(p)$ is the density dependent conductivity, $p_{c}$ is the critical density, and $\delta$ is the critical exponent descibing the transition. $A$ is a constant of proportionality. The $2 \mathrm{D}$ percolation exponent is expected to be 1.31 [1], 21, 22]. The results of such an analysis applied to our sample is shown in Figure 4(b). The extracted exponent for our sample is $\delta=1.7 \pm 0.1$ and the critical density $p_{c}=0.38 \times 10^{10} \mathrm{~cm}^{-2} \pm 0.01 \times 10^{10} \mathrm{~cm}^{-2}$. Formally, the preceding analysis is valid for $\left(p-p_{c}\right) \ll p_{c}$. While the quality of the fit does not depend sensitively on the number of data points included, clearly the restriction $\left(p-p_{c}\right) \ll p_{c}$ must be relaxed somewhat to make connection with the experimental data. Interestingly, the extracted conductivity parameters from the percolation analysis are consistent with known behavior of very high mobility n-type GaAs 2D systems as reported in ref. [11]. In particular, our extracted percolation exponent of 1.7 at $T=47 \mathrm{mK}$ is almost identical with the corresponding exponent value at a somewhat higher temperature $(\sim 100-200 \mathrm{mK})$ in ref. [11], which is consistent with equivalent values of the dimensionless temperature $T / T_{F}$ for the two systems, where $T_{F}$ is the Fermi temperature, and our critical density of $3-4 \times 10^{9}$ $\mathrm{cm}^{-2}$ is consistent with the corresponding critical electron density in ref. [11]. The temperature dependences of the fit parameters are detailed in figure 5. To conclude, we find that the temperature dependence of the metallic phase is well-described by assuming the conductivity to be limited by temperature and density dependent screened charged impurity scattering whereas the apparent low-density $\left(\sim 3.8 \times 10^{9} \mathrm{~cm}^{-2}\right)$ transition from the metal to the insulating phase is well-described by a density-inhomogeneity driven percolation transition.

[1] E. Abrahams, P. W. Anderson, D. C. Licciardello, and T. V. Ramakrishnan, Phys. Rev. Lett. 42, 673 (1979)

[2] S. V. Kravchenko, G. V. Kravchenko, J. E. Furneaux, V. M. Pudalov, and M. D'Iorio, Phys. Rev. B 50, 8039 (1994).

[3] E. Abrahams et al., Rev. Mod. Phys. 73, 251 (2001); S. V. Kravchenko and M. P. Sarachik, Rep. Prog. Phys. 67, 1 (2004).

[4] S. Das Sarma and E. H. Hwang, Solid State Comm. 135, 579 (2005).

[5] A. Punnoose and A. M. Finkel'stein, Science 310, 289 (2005).

[6] G. Zala, B. N. Narozhny, and I. L. Aleiner, Phys. Rev. B 64, 214204 (2001)

[7] K. Lai, W. Pan, D. C. Tsui, S. A. Lyon, M. Muhlberger, and F. Schaffer, Phys. Rev. B, 72, R081313 (2005)

[8] S. Anissimova, S. V. Kravchenko, A. Punnoose, A. M. Finkel'stein, and T. M. Klapwijk, Nature Physics, 3, 707 (2007)

[9] J. Zhu et al., Phys. Rev. Lett. 90, 056805 (2003).

[10] M. P. Lilly et al., Phys. Rev. Lett. 90, 056806 (2003).

[11] S. Das Sarma, M. P. Lilly, E. H. Hwang, L. N. Pfeiffer, K. W. West, and J. L. Reno, Phys. Rev. Lett. 94, 136401 (2005).

[12] J. Yoon, C. C. Li, D. Shahar, D. C. Tsui, and M. Shayegan, Phys. Rev. Lett. 821744 (1999)

[13] M. Y. Simmons et al., Phys. Rev. Lett. 80, 1292 (1998)

[14] G. Allison et al. Phys. Rev. Lett. 96216407 (2006)

[15] L. Tracy et al., Solid State Commun. 137, 150 (2006).

[16] M. J. Manfra, L. N. Pfeiffer, K. W. West, R. de Picciotto, and K. W. Baldwin, Appl. Phys. Lett. 85, 162106 (2005).

[17] H. Zhu, K. Lei, D. C. Tsui, S. P. Bayrakci, N. P. Ong, M. J. Manfra, L. N. Pfeiffer, and K. W. West, Solid State Commun. 141, 510 (2007).

[18] A. P. Mills, Jr., A. P. Ramirez, L. N. Pfeiffer, and K. W. West, Phys. Rev. Lett. 83, 2805 (1999)

[19] H. Noh et al. Phys. Rev. B 68, R241308 (2003)

[20] S. Das Sarma and E. H. Hwang, Phys. Rev. B 68, 195315 (2003).

[21] R. Leturcq et al., Phys. Rev. Lett. 90, 076402 (2003); Y. Hanein et al., Phys. Rev. B 58, R7520 (1998); Y. Meir, Phys. Rev. Lett. 83, 3506 (1999).

[22] A. L. Efros, Solid State Commun. 65, 1281 (1988); 70, 253 (1989); S. He and X. C. Xie, Phys. Rev. Lett. 80, 3324 (1998).

[23] S. Das Sarma and E. H. Hwang, Phys. Rev. B 69, 195305 (2004). 